\title{
An x-ray fluorescence imaging system for gold nanoparticle detection
}

\author{
K Ricketts ${ }^{1}$, C Guazzoni ${ }^{2}$, A Castoldi ${ }^{2}$, A P Gibson ${ }^{1}$ and G J Royle ${ }^{1}$ \\ ${ }^{1}$ Department of Medical Physics and Bioengineering, University College London, UK \\ ${ }^{2}$ Dipartimento di Elettronica e Informazione, Politecnico di Milano, Italy and INFN, \\ Sezione di Milano, Italy \\ E-mail:
}

Received 26 February 2013, in final form 23 September 2013

Published DD MMM 2013

Online at stacks.iop.org/PMB/58/1

\begin{abstract}
Gold nanoparticles (GNPs) may be used as a contrast agent to identifytumour location and can be modified to target- and image-specific tumour biological parameters. There are currently no imaging systems in the literature that have sufficient sensitivity to GNP concentration and distribution measurement at sufficient tissue depth for use in in vivo and in vitro studies. We have demonstrated that high detecting sensitivity of GNPs can be achieved using $\mathrm{x}$-ray fluorescence; furthermore this technique enables higher depth imaging in comparison to optical modalities. Two X-ray fluorescence systems were developed and used to image a range of GNP imaging phantoms. The first system consisted of a $10 \mathrm{~mm}^{2}$ silicon drift detector coupled to a slightly focusing polycapillary optic which allowed 2D energy resolved imaging in step and scan mode. The system has sensitivity to GNP concentrations as low as $1 \mathrm{ppm}$. GNP concentrations different by a factor of 5 could be resolved, offering potential to distinguish tumour from non-tumour. The second system was designed to avoid slow step and scan image acquisition; the feasibility of excitation of the whole specimen with a wide beam and detection of the fluorescent $\mathrm{x}$-rays with a pixellated controlled drift energy resolving detector without scanning was investigated. A parallel polycapillary optic coupled to the detector was successfully used to ascertain the position where fluorescence was emitted. The tissue penetration of the technique was demonstrated to be sufficient for nearsurface small-animal studies, and for imaging 3D in vitro cellular constructs. Previous work demonstrates strong potential for both imaging systems to form quantitative images of GNP concentration.
\end{abstract}

(Some figures may appear in colour only in the online journal)

\section{Introduction}

Nanoparticles (NPs) may be used as a contrast agent to identify tumours; a combination of leaky vasculature and poor lymphatic drainage around tumour sites (an effect referred to as 
enhanced permeability and retention) results in increased NP uptake by tumour cells compared to healthy cells (Hainfeld et al 2011). Furthermore, NPs can be attached to antibodies to actively target key tumour characteristics in order to inform treatment options and prognosis (Popovtzer et al 2008, Jiao et al 2011).

Gold NPs (GNPs) have readily modifiable surfaces to increase solubility (in order to travel through the bloodstream) and enhance cell-specific uptake (Giljohann et al 2010). GNP coating with polyethylene glycol (PEG), sodium citrate or heparin lowers acute toxicity and sufficiently evades both host immune reactions and irreversible binding to circulating serum proteins (Kemp and Linhardt 2010). Their high atomic number makes them an ideal contrast medium for use in x-ray imaging techniques (Chen et al 2013, Hainfeld et al 2012, Kuang et al 2013). Molecular imaging is also possible due to the greater number of atoms delivered to the receptor site ( $\sim 250 \mathrm{Au}$ atoms per $1.9 \mathrm{~nm} \mathrm{NP}$ compared with three (monomer) or six (dimer) I atoms per molecule when using iodinated contrast agents (Hainfeld et al 2006), thereby increasing the signal per cell. A great advantage of using GNPs as an imaging contrast agent for radiotherapy is that GNPs have been shown to enhance local dose deposition for both X-ray radiotherapy (Hainfeld et al 2010, 2012, Zhang et al 2009, Jain et al 2011) and proton radiotherapy (Kim et al 2010).

There has been a growing interest in functionalizing NPs to better target cancer treatment and imaging (Hainfeld et al 2004, 2011, Sokolov et al 2004, Jain et al 2012, McMahon et al 2012, Mccarthy and Weissleder 2009). These studies primarily use in vivo small-animal studies and correlate treatment outcomes with the amount and location of NPs. Other studies investigate uptake and distribution of NPs in 3D in vitro cellular structures, such as cellular hydrogels and multicellular tumour spheroids (Goodman et al 2009, Grainger et al 2010); the thickness of such in vitro samples are reported to range from 1-15 mm. It has previously been noted by Cheong et al (2010) that the following two technical challenges are yet to be met satisfactorily by such studies: (i) imaging of GNPs distributed within the tumour and other critical organs, and (ii) quantification of the amount of GNPs present. We propose an additional important challenge that must be met for GNP imaging systems: (iii) achieving a system sensitivity capable of detecting GNP concentrations typically found in tumours. This sensitivity will depend on tumour type, organ, stage and targeted biomarker expression. A study that imaged the distribution of functionalized (with Herceptin) and unfunctionalized GNP uptake in mice showed an increased uptake of the GNPs in Her2+ tumours compared with Her2- tumours, with typical functionalized GNP concentrations ranging from 1-4 mgAu ml ${ }^{-1}$ in tumours. A high dose of GNPs $\left(1.1 \mathrm{mgAu} \mathrm{g}^{-1}\right)$ was injected into the mouse, but other studies presented in the literature administer a much lower dose of typically $0.01 \mathrm{mgAu} \mathrm{g}^{-1}$ (Khlebtsov and Dykman 2011). In order for use in early stage cancer detection a technique sensitive to less than this is required. Tumour/muscle contrast values were also presented by Hainfeld et al (2011) with whole tumour/muscle and tumour periphery/muscle values for functionalized (unfunctionalized) GNPs of 11.4 (8.5) and 21.8 (13.9) respectively.

A range of non-invasive GNP imaging solutions have been presented in the literature, but none so far have met all requirements set out above. X-ray fluorescence CT (XFCT) imaging of GNP-loaded phantoms has been successfully performed using K-X-ray fluorescence (K-XRF) in gold (emission energies of 67 and $68.8 \mathrm{keV}$ ) (Cheong et al 2010). However, relatively high GNP concentrations were tested by the system, the lowest being $5 \mathrm{mgAu} \mathrm{ml}^{-1}$ (three orders of magnitude greater than that expected in vivo). Hainfeld et al were the first group to image GNPs in vivo in mice using microCT; the mice were injected intravenously with $1.9 \mathrm{~nm}$ GNPs at a concentration of $2.7 \mathrm{mgAu} \mathrm{g}^{-1}$ (Chen et al 2013). Deliberately high amounts of gold were used to investigate x-ray contrast given by the NPs. Another group have demonstrated that good contrast to noise CT images could be obtained at gold concentrations 
of $0.1 \mathrm{mgAu} \mathrm{m}^{-1}$ (Dilmanian et al 1997). A more recent study by Hainfeld et al (2011) reports that microCT enables quantification of GNPs within various regions of a murine tumour; a calibrated relationship between radiodensity and gold concentration was demonstrated down to $0.05 \mathrm{mgAu} \mathrm{ml} \mathrm{m}^{-1}$. Techniques based on optical spectroscopy were found to be satisfactory for the quantification of GNP concentration in vivo (Zaman et al 2007, Qian et al 2008), but their use is limited to shallow sites ( $\sim 5 \mathrm{~mm}$ deep). Photo-acoustic imaging (Yang et al 2009) and surface-enhanced Raman spectroscopy techniques (Keren et al 2008) have also been used to image GNPs, along with multi-modal imaging techniques (Kircher et al 2012).

There is currently a need for an in vivo system that can penetrate to greater depths than the aforementioned optical techniques and that is sensitive to even lower GNP concentrations than the techniques mentioned above. We have developed a GNP imaging system based on L$\mathrm{X}$-ray fluorescence (L-XRF) that meets the required technical challenges outlined above. It has previously been demonstrated that the system is capable of quantitative GNP concentration measurement down to the system detection limit of $0.001 \mathrm{mgAu} \mathrm{ml}^{-1}$ (Dilmanian et al 1997); to the authors' knowledge this sensitivity is unparalleled by any other system present in the literature. GNP detection criteria (ii) and (iii) has therefore already been demonstrated for this system (Dilmanian et al 1997), and so this work reports translation of the developed 1D L-XRF spectroscopic system to a 2D imaging system to demonstrate ability to meet criteria (i). Two proposed systems were developed and used to image a range of GNP imaging phantoms; one consisting of a scanning spectroscopic module, the other consisting of an energy resolving pixellated detector.

\section{Materials and methods}

\subsection{Nanoparticle imaging phantom}

A GNP imaging phantom was designed in order to assess the ability of the $\mathrm{x}$-ray fluorescence (XRF) imaging system to resolve different GNP concentrations. A concentric ring configuration was selected, enabling one GNP concentration to be surrounded by a GNP solution of different concentration. The phantom consisted of a series of $9 \mathrm{~mm}$ holes drilled into a $10 \mathrm{~mm}$ thick Perspex slab; an inner hole of diameter $5 \mathrm{~mm}$ was drilled in the centre of the $9 \mathrm{~mm}$ hole to contain a hollow cylinder of $5 \mathrm{~mm}$ diameter. This acted as a barrier between the two GNP concentration solutions.

Two concentration pairings were selected: (i) a high contrast phantom consisted of inner (outer) GNP concentrations 4 (0.2) $\mathrm{mgAu} \mathrm{ml}^{-1}$, mimicked the difference in Herceptinfunctionalized GNP uptake in tumour periphery and muscle (20:1 contrast), at exaggerated GNP concentration levels found in the Hainfeld et al (2011) mouse study, (ii) a low contrast phantom consisted of inner (outer) GNP concentrations of $0.125(0.025) \mathrm{mgAu} \mathrm{ml}^{-1}$ mimicked unfunctionalized GNP uptake in whole tumour and muscle (5:1 contrast) at lower GNP concentrations reported in in vivo and in vitro studies (Khlebtsov and Dykman 2011). The outer concentration was selected to be lower than the inner concentration in each case to mimic the in vivo situation of greater uptake in the tumour compared with uptake in surrounding tissues.

\subsection{Step and scan system}

The step and scan system consisted of a $10 \mathrm{~mm}^{2}$ silicon drift detector (SDD) coupled to a slightly focusing polycapillary optic which allowed 2D mapping in step and scan mode. Imaging was performed at the Diamond Synchrotron Source, Didcot, UK. A laminar X-ray 

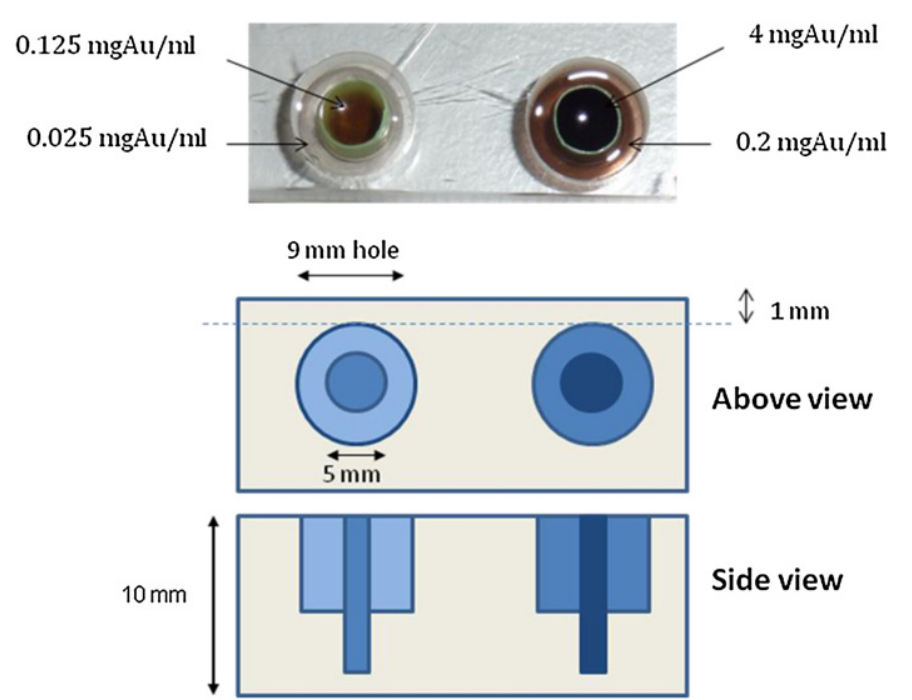

Figure 1. Contrast phantom; $9 \mathrm{~mm}$ hole including a straw located centrally to provide a barrier between two different GNP concentration solutions. Right detail: high contrast phantom filled with GNP solutions $4 \mathrm{mgAu} \mathrm{ml}^{-1}$ (central) and $0.2 \mathrm{mgAu} \mathrm{ml}^{-1}$ (outer ring); left detail: low contrast phantom filled with GNP solutions $0.125 \mathrm{mgAu} \mathrm{ml}^{-1}$ (central) and $0.025 \mathrm{mgAu} \mathrm{m}^{-1}$ (outer ring).

beam irradiated the sample close to the sample surface (the top edge of the beam set to the sample meniscus) to minimize self-attenuation of the fluorescence signal.

The photoelectric cross section for XRF is greatest when the exciting $\mathrm{x}$-ray is just above the absorption edge for the considered shell. The beamline energy was set to $15 \mathrm{keV}$ (above the edge for gold L-fluorescence of $11.9 \mathrm{keV}$ and $13.7 \mathrm{keV}$ for $\mathrm{L} \alpha / \mathrm{L} \beta_{2}$ and $\mathrm{L} \beta_{1}$ fluorescence respectively). The energy set was $1.3 \mathrm{keV}$ above the highest L-edge of gold, a compromise between optimum excitation and minimization of the impact of the Compton shoulder background on the gold fluorescence line. The beamline collimators were set to give a beam $4 \mathrm{~mm}$ wide and $4 \mathrm{~mm}$ high, with the top of the beam in line with the meniscus of the sample. No filtration was added to the beam.

The SDD detection module was mounted with a $90^{\circ}$ geometry in order to minimize the Compton background, and positioned on the $z$-axis in line with the centre of the GNP inclusions of the phantom. A CCD camera located behind the sample was used to monitor the beam-sample alignment. An ionization chamber was used to monitor beam intensity in order to normalize the data. The experimental set-up of the $2 \mathrm{D}$ scanning system making use of a focusing polycapillary optic is displayed in figure 2 .

2.2.1. X-ray detector system. A $10 \mathrm{~mm}^{2} \mathrm{SDD}$ chip, produced in polysilicon technology by PNSensor GmbH and featuring an onchip JFET, was mounted in a dedicated vacuum-tight housing together with a Peltier cooler and dedicated front-end electronics in charge amplifier configuration with pulsed reset developed by Politecnico di Milano, Italy, and INFN Milano (Alberti et al 2009). The measured energy resolution was $138 \mathrm{eV} \mathrm{FWHM} \mathrm{at} \mathrm{a} \mathrm{shaping} \mathrm{time}$ of $1 \mu \mathrm{s}$ at $5.9 \mathrm{keV}$ and a maximum count rate of the order of $105 \mathrm{~s}^{-1}$. The SDD consisted of a $450 \mu \mathrm{m}$ thick detector-grade silicon substrate, and gave a high efficiency of gold L $\alpha$ peak detection $(97 \%)$.

The preamplifier signal was fed to a fifth-order shaping amplifier with $1 \mu$ s shaping time constant (a measured value of $134 \mathrm{eV} \mathrm{FWHM}$ at $5.9 \mathrm{keV}$ ). A multichannel analyser 

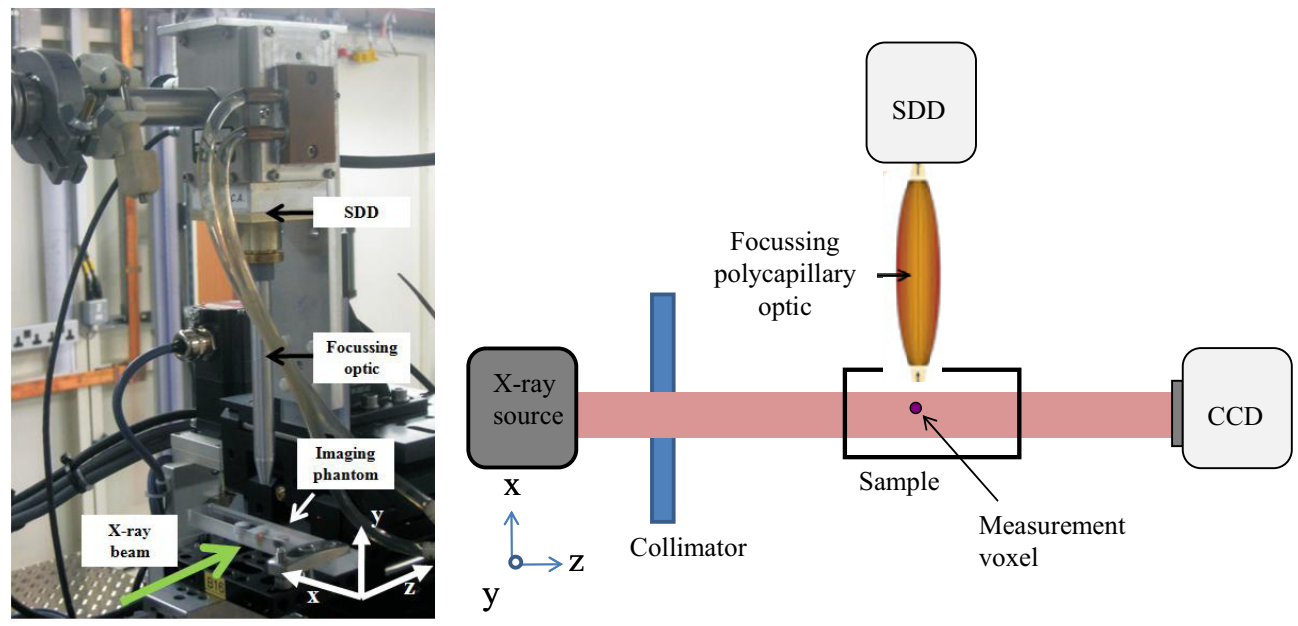

Figure 2. Experimental set-up for 2D scanning system.

(MCA-8000A, Amptek Inc., MA, USA) interfaced the detector with a personal computer for data acquisition and spectrum analysis.

2.2.2. Focusing polycapillary optics. Polycapillary lenses are monolithic structures of bundles of hollow glass capillaries along which x-rays are guided by total external reflection (TER) (MacDonald and Gibson 2003, Kumakhov 1990). Focusing polycapillary optics are generally used to capture $\mathrm{x}$-rays emitted from a source and focus them to a small spot area to increase the intensity over small sample areas to enable high intensity XRF measurement. Focusing optics are designed to have gently bending hollow capillaries of curvature smaller than the critical angle that propagate $\mathrm{x}$-rays through them to focus them to a point. According to the physics of $\mathrm{x}$-ray optics smaller focal spot sizes are expected with increasing $\mathrm{x}$-ray energy (Grassi et al 2010).

The XRF imaging system described here utilized a focusing polycapillary optic to collect the X-rays emitted from the sample after XRF excitement, and to transport the collected $\mathrm{x}$-rays to the detector chip. The lens enabled an increased distance between the sample and detector without a decrease in effective collection angle of the XRF x-rays. The lens also improved the spatial resolution of the image by allowing measurement of small areas of the sample, with particular improvements made compared with mechanical collimator techniques. The focusing lens also enabled the detector to focus measurement at a specific depth in the sample (along the $y$-axis) according to the focal point of the lens. An 84MLS11 polycapillary optic lens (Institute for Scientific Instruments GmbH, Berlin, Germany) (figure 3) was implemented and measured to have a nominal focusing spot size of $\sim 300 \mu \mathrm{m}$ FWHM in the energy range 5-10 keV (Grassi et al 2010).

The detector was sealed in a custom compact case, vacuum-tight down to $10^{-4}$ mbar. The housing was sealed with an $8 \mu \mathrm{m}$ thick beryllium entrance window (Castoldi et al 2011). Lens parameters given by the manufacturer are as follows: capture angle 0.095 rad; capillary diameter $30 \mu \mathrm{m}$; lens length $85 \mathrm{~mm}$; lens curvature radius $1050 \mathrm{~mm}$.

2.2.3. Gold nanoparticle imaging procedure. The beam, sample centre and SDD-optic module were aligned. The sample was then scanned in both the $x$ - and $z$-directions with a $600 \mu \mathrm{m} \times 600 \mu \mathrm{m}$ pitch to build up a pixellated image. The scanned area was 

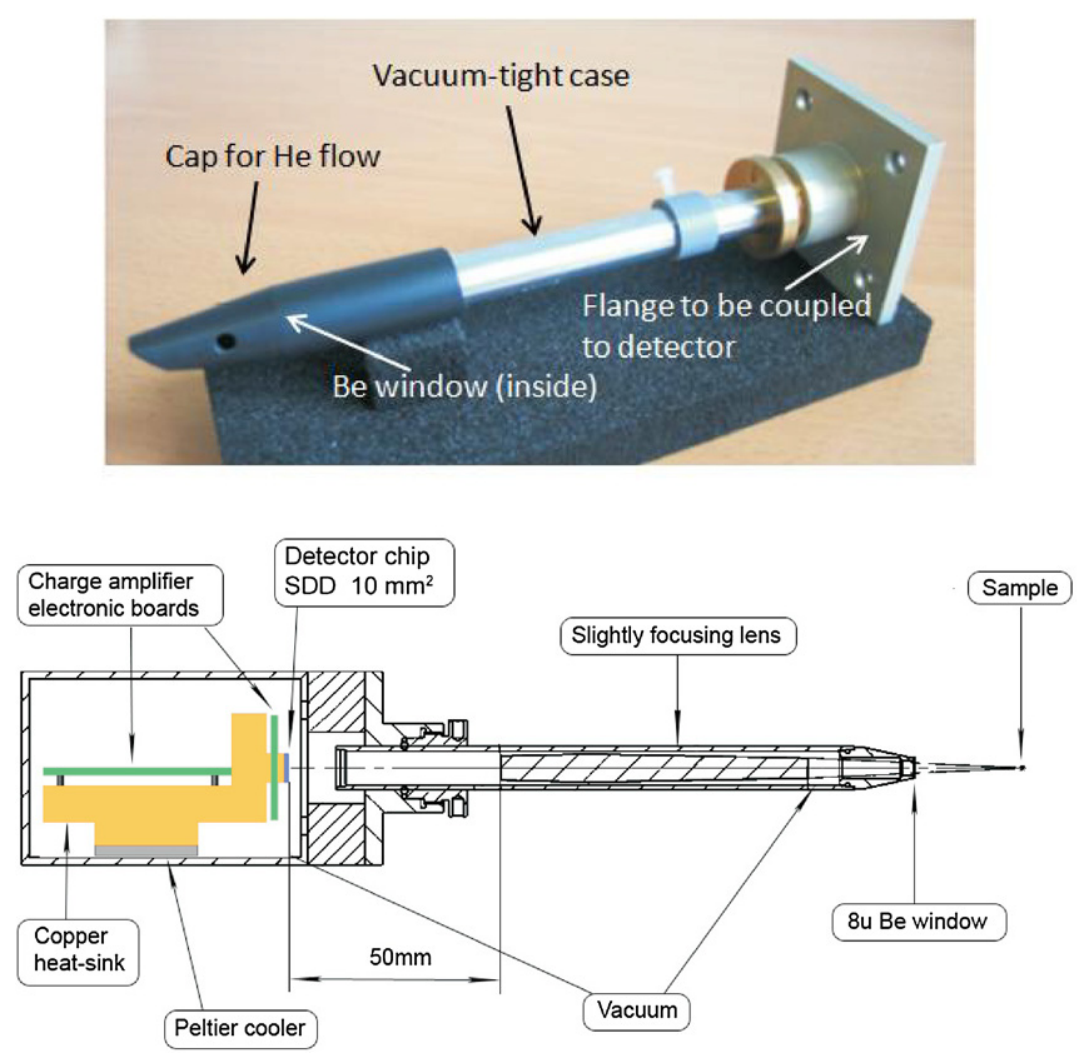

Figure 3. (Top) 84MLS11 polycapillary optic lens (Institute for Scientific Instruments GmbH, Berlin, Germany) in its vacuum-tight case. (Bottom) Designed vacuum-tight detection module with the polycapillary lens (Keren et al 2008).

$12 \mathrm{~mm} \times 7.2 \mathrm{~mm}$. An X-Y-Z micrometric stage system was responsible for sample movement and remote control management by the DAQ enabled automatic $\mathrm{X}-\mathrm{Y}$ scans. An acquisition time of $40 \mathrm{~s}$ per point was taken (total imaging time $3 \mathrm{~h}$ ) and the full energy spectrum obtained at each point.

GNP images were reconstructed off-line by computing the integral counts under the gold $\mathrm{L} \alpha$ and $\mathrm{L} \beta$ peaks. Figure 4 displays example energy spectra within the Perspex component of the phantom, and within the $4 \mathrm{mgAu} \mathrm{ml}^{-1} \mathrm{GNP}$ solution volume. It can be seen that the background was negligible beneath the gold peaks, and so no background subtraction was made when integrating the gold peak counts.

Radiation scattered by the sample can be used as an internal standard to correct for the effects of self-absorption and geometrical variations on measured XRF signal (Andermann and Kemp 1958, Bui et al 1989). We have previously demonstrated the usefulness of this technique for this application; the accuracy of concentration measurement was found to improve by $71 \%$ on performing the scatter correction (Ricketts et al 2012). Correction involved normalizing to the image obtained using the integral counts of a region of interest covering the coherent scatter peak of each spectrum. The tails of the elastic scattering peak and of the Compton scattering peaks show an overlap that was neglected in this analysis. It should be noted that this normalization technique is only appropriate if the scatter signal is sufficiently large such that its Poisson statistics do not worsen the accuracy of concentration measurement. 

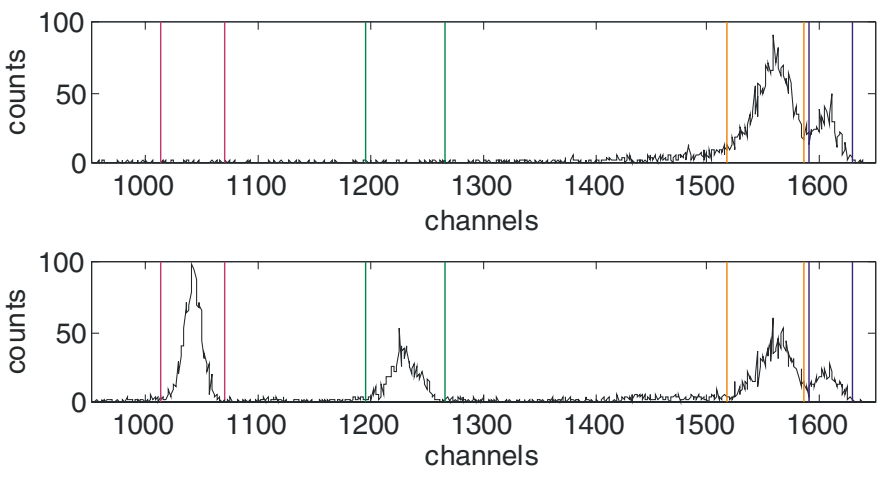

Figure 4. Sample energy spectra collected in two different points of the scan in pure Perspex (top) and within the hole filled with $4 \mathrm{mgAu} \mathrm{ml}^{-1} \mathrm{GNP}$ solution (bottom). Regions of interest used for the computation of the integral counts are included on the spectra-from left to right: gold L $\alpha$ line, gold $\mathrm{L} \beta$ line, Compton scattering peak and elastic scattering peak.

The image reconstruction protocol did not attempt to quantify GNP concentration in each pixel, and was undertaken to demonstrate the feasibility of forming an image of GNP distribution. GNP concentration measurement has been previously demonstrated (Ricketts et al 2012), and this approach collected the same information but from a smaller volume, and therefore the ability to quantify GNP concentrations and the system detection limit should remain unchanged if sufficient counting times are used.

\subsection{One-shot system}

In order to avoid step and scan XRF imaging techniques which potentially require long measurement times, the feasibility of excitation of the whole sample with a wide beam and detection of the XRF $\mathrm{x}$-rays with a $2 \mathrm{D}$ energy resolving pixellated controlled drift detector (CDD) has been investigated. Use of a collimating polycapillary optic coupled to the detector preserves the positional information from where fluorescence was emitted. Measurements were made at the SYRMEP beamline at the Elettra synchrotron source, Trieste, Italy. The synchrotron source energy was set to $15 \mathrm{keV}$. A beam size was set to completely cover the detection area of the sample (detector dimensions $4.3 \mathrm{~mm} \times 6.1 \mathrm{~mm}$ ). The sample thickness measured was $3.8 \mathrm{~mm}$, and the view area limited to the field of view of the polycapillary optic lens and the detector area.

The imager was mounted with a $90^{\circ}$ geometry and coupled with a parallel polycapillary optic to ascertain the position of emission of gold fluorescence. A CCD camera behind the sample monitored the beam-sample alignment and made conventional transmission images to assist beam-sample-detector alignment. An ionization chamber was used to monitor beam intensity. The experimental set-up is displayed in figure 5.

2.3.1. X-ray detector system. A CDD (Politecnico di Milano, INFN Milano and MPI Munich) of pixel size $180 \mu \mathrm{m} \times 180 \mu \mathrm{m}$, and 24 drift channels each with 34 pixels was used to form the XRF images. The CDD was coupled to a collimating polycapillary optic angular filter (IfG Berlin), of channel size $30 \mu \mathrm{m}$.

The CDD was a fully depleted silicon detector that operated in single-photon counting integrate-readout mode and allowed detection of position and energy of each photon in the range $0.5-30 \mathrm{keV}$. High frame rates (up to $100 \mathrm{kHz}$ ) were achievable (Castoldi et al 


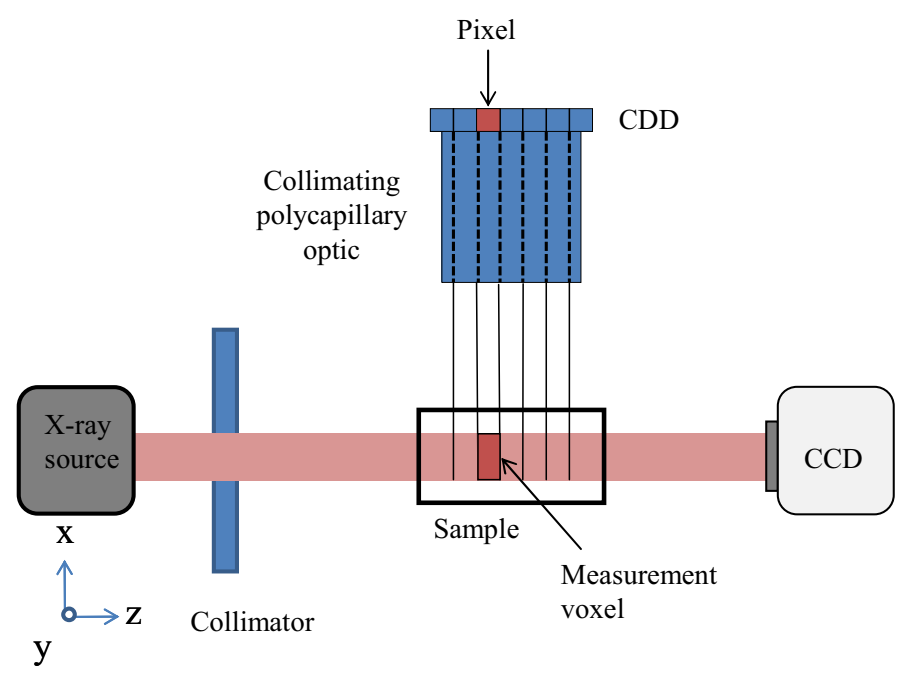

Figure 5. Experimental set-up of CDD XRF imaging system.

2007). The chip thickness was $300 \mu \mathrm{m}$ allowing absorption efficiency of $93 \%$ for the gold $\mathrm{L} \alpha$ line. The measured energy resolution was $320 \mathrm{eV}$ at the manganese $K \alpha$ line $(5.9 \mathrm{keV})$. During measurement at Elettra, the energy resolution was found to degrade to $460 \mathrm{eV} \mathrm{FWHM}$ measured at the copper $K \alpha$ line $(8 \mathrm{keV})$, due to the noisy environment of a synchrotron beam.

Equally spaced potential wells were generated along the length of the detector; the charge generated by the interacting photon was collected in these wells. The potential barriers were then removed and the charge collected drifted towards the readout located at the end of each column. One positional coordinate was given by drift time, the other by knowing which drift column the event occurred. The amplitude of the signal pulse provided the energy information.

The characteristics of both the CDD and SDD systems have been compared in table 1 .

2.3.2. Collimating polycapillary optics. In order to acquire a 2D XRF image of the sample on the detector in one shot, a detector collimator was required. The spatial resolution of an XRF image is a summation in quadrature of the detector pixel size and the collimator hole size. A multi-hole mechanical collimator (typically with hole dimensions limited to $\sim 500 \mu \mathrm{m}$ ) may have been used. However, the hole size would have dominated the spatial resolution. To overcome the practical limitations of mechanical collimator hole size polycapillary optic technology was used; a parallel polycapillary optic (model 72MLS) made available by IfG $\mathrm{GmbH}$, Berlin, was implemented with glass capillary channel diameter $30 \mu \mathrm{m} \pm 1 \mu \mathrm{m}$, bunched together in parallel to form a monolithic structure of total diameter $8 \mathrm{~mm}$. The capillaries were $40 \mathrm{~mm}$ in length. The capillaries were encased in a metal cylinder of outer diameter $12 \mathrm{~mm}$ and total length $44 \mathrm{~mm}$.

$\mathrm{X}$-rays were only accepted into the optic channels if the incident angle of the X-ray was below the critical angle for TER at that energy $\left(\theta_{c}(E)\right)$. The proposed system used an optic made of borosilicate glass, for which $\theta_{c}(E)$ is:

$$
\theta_{c}(E)=\frac{30}{E} \operatorname{mrad}
$$

where $E$ is the photon energy in $\mathrm{keV}$ (Wang et al 1996). 
Table 1. Characteristics of the SDD and CDD systems for use in XRF imaging. The image pixel size of the CDD was calculated as a quadrature sum of CDD pixel size and polycapillary optic channel size.

\begin{tabular}{lll}
\hline Parameter & SDD + focusing optic & CDD + collimating optic \\
\hline Energy resolution & $350 \mathrm{eV}$ at $20 \mathrm{keV}$ & $1 \mathrm{keV}$ average at 20 keV \\
Max count rate & $100 \mathrm{kHz}$ & $100 \mathrm{kHz}$ \\
Substrate thickness & $450 \mu \mathrm{m} \mathrm{Si}$ & $300-450 \mu \mathrm{m} \mathrm{Si}$ \\
Efficiency at L $\alpha$ & $97 \%$ & $93-97 \%$ \\
Image pixel size & $300 \mu \mathrm{m}$ (focus spot size) & $182 \mu \mathrm{m}$ \\
\hline
\end{tabular}

$\theta_{c}(E)$ was $<5$ mrad for $\mathrm{x}$-rays in the 5-30 keV energy range, and the fibres therefore acted as fine angular collimators, filtering through $\mathrm{x}$-rays at the selected scatter angle and rejecting $\mathrm{x}$-rays incident at all other angles. This allowed formation of a 2D image (across the area of the optic) of the X-rays emitted in the solid angle of collection of the optic, and therefore enabled faster image acquisition than point-by-point scanning. Optic channel sizes of the order of a few tens of micrometres meant that the detector pixel size (180 $\mu \mathrm{m}$ for the CDD) set the limiting factor for the spatial resolution. Use of a parallel optic improved the spatial resolution by a factor of $\sim 2.5$ compared with using a typical multi-hole collimator (from $\sim 500 \mu \mathrm{m} 10$ $\sim 180 \mu \mathrm{m}$ ).

It should be noted that the optic cannot be employed at high $\mathrm{x}$-ray energies (such as for $\mathrm{K}-\mathrm{XRF}$ techniques) due to penetration of x-rays through the glass fibres; a collimator with different glass composition could be designed with high $Z$ components for use at high energies. The use of the current optic is limited to application in L-XRF techniques.

2.3.3. Gold nanoparticle imaging procedure. The beam, detector and sample were aligned. The sample image area was fully illuminated by the synchrotron beam, and the CDD set to acquire for $6500 \mathrm{~s}$ for the high contrast phantom and $11000 \mathrm{~s}$ for the low contrast phantom. Regions of interest were set over the gold $\mathrm{L} \alpha$, gold $\mathrm{L} \beta$ and coherent scatter peaks for each detector pixel spectrum. The latter region of interest was set to perform a scatter normalization to the XRF image to correct for sample absorption.

\subsection{Measurements at depth}

The aim of this technique is to image GNP concentrations in vivo or in thick ex vivo samples, and so the effect of overlying tissue on the XRF signal must be determined. The L-XRF system was used to perform a series of XRF measurements of an $8 \mathrm{mgAu} \mathrm{ml}^{-1} \mathrm{GNP}$ solution suspended in de-ionized water with five different thicknesses of Perspex $(0,2,4,8$ and $16 \mathrm{~mm}$ ) placed between the sample and detector (see figure 6). Perspex has similar attenuating properties to tissue at these energies. Each measurement was acquired for $180 \mathrm{~s}$.

\section{Results}

\subsection{Step and scan system}

Figure 7 displays the 2D pixellated images obtained by plotting the logarithm of the gold $\mathrm{L} \alpha$ and $\mathrm{L} \beta$ peak ROIs as a function of scan position for the high contrast phantom (with and without normalizing to the coherent scatter peak), and also the scatter normalized low concentration phantom. The primary beam is incident on the right side of the images in figure 7 . 


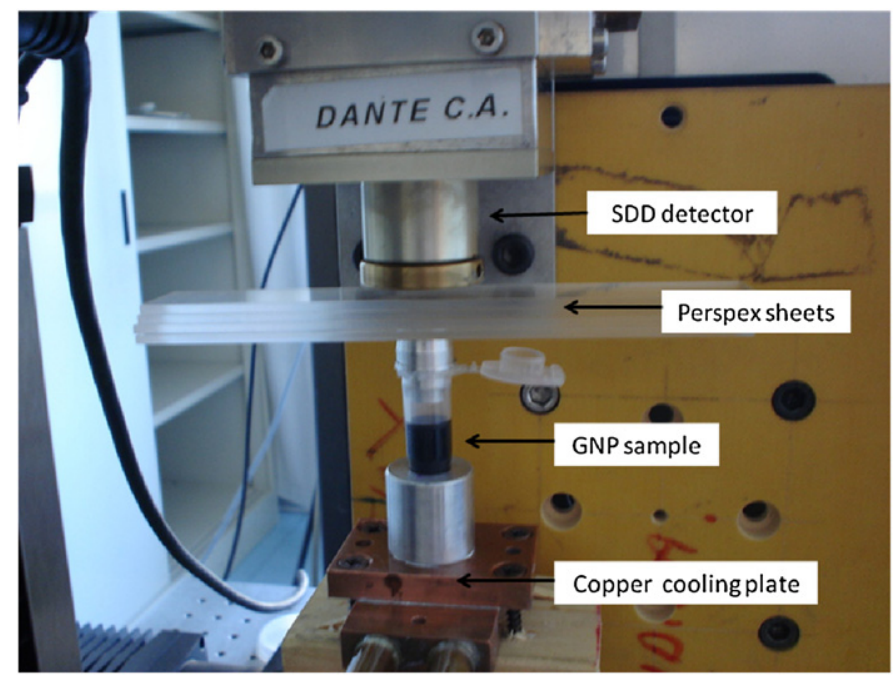

Figure 6. Depth measurement of $8 \mathrm{mgAu} \mathrm{ml}^{-1} \mathrm{GNP}$ sample; a range of Perspex thicknesses were added above the sample to test the effect of depth of measurement on the L-XRF method.
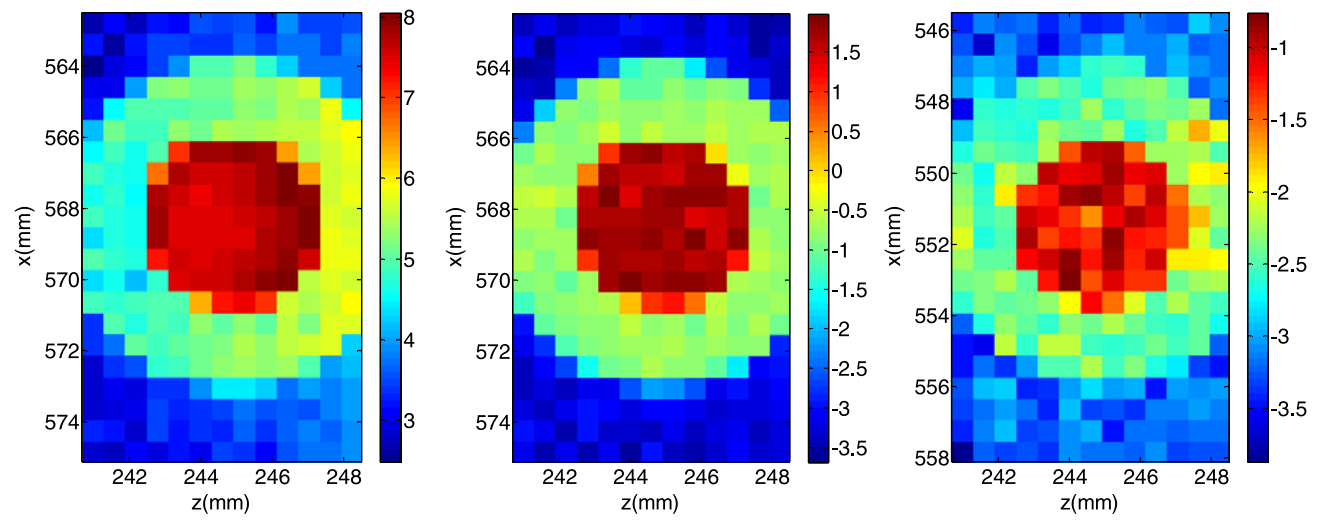

Figure 7. 2D gold XRF images in log scale of GNP contrast phantoms acquired using an SDD coupled to a focusing polycapillary optic. The primary beam is incident on the right-hand side of the images. (Left) Un-normalized high contrast phantom, (middle) scatter normalized high contrast phantom, and (right) coherent scatter normalized low concentration phantom.

\subsection{One-shot system}

Two XRF images of a high contrast and low contrast phantom are displayed in figure 8.

The high contrast image in figure 8 displays presence of an iron slab alignment tool as a cold spot on the image (i.e. very few X-rays were detected in the gold ROI at the position of the iron). A cold spot is also present on the bottom of the low contrast image due to the location of the lens border.

\subsection{Measurements at depth}

The effect of adding Perspex thickness to the measured XRF spectra is displayed in figure 9. 

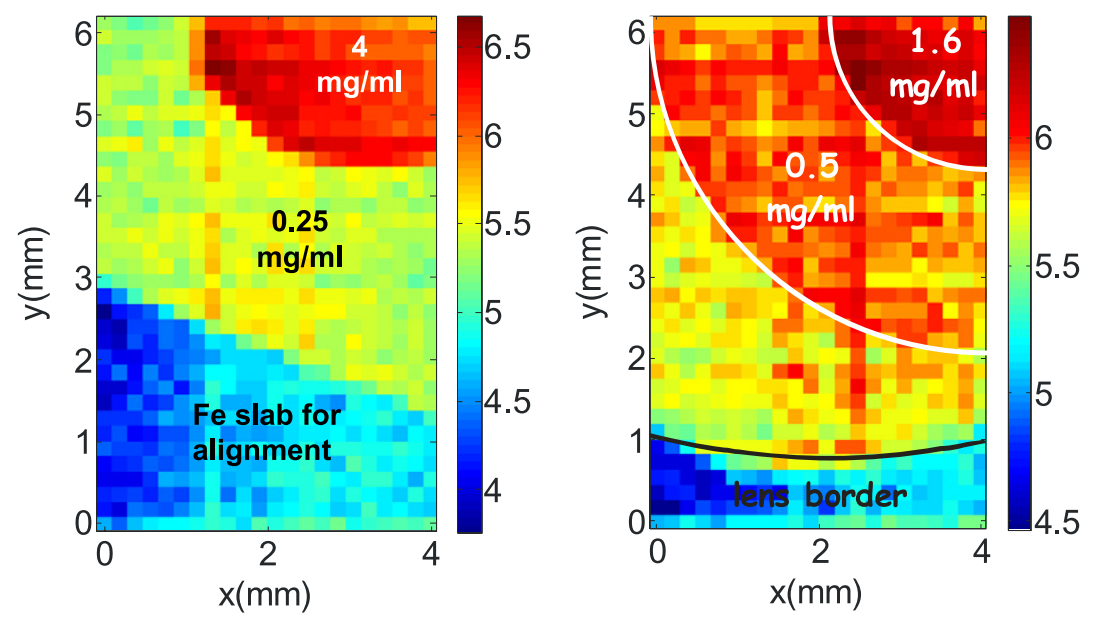

Figure 8. XRF images (log scale) of GNP contrast resolution phantom using the CDDpolycapillary optic XRF system. The images are of high contrast (left) and low contrast (right) details. GNP concentrations are labelled on the images.

Table 2. Predicted and measured attenuation of gold fluorescence signals through varying thicknesses of Perspex.

\begin{tabular}{lll}
\hline $\begin{array}{l}\text { Perspex } \\
\text { thickness }(\mathrm{mm})\end{array}$ & $\begin{array}{l}\text { Measured (predicted) } \\
\text { gold } \mathrm{L} \alpha \text { reduction } \%\end{array}$ & $\begin{array}{l}\text { Measured (predicted) } \\
\text { gold } \mathrm{L} \beta \text { reduction } \%\end{array}$ \\
\hline 2 & $54(60)$ & $38(47)$ \\
4 & $77(84)$ & $61(72)$ \\
8 & $95(97)$ & $84(92)$ \\
16 & $99.2(99.9)$ & $89(99)$ \\
\hline
\end{tabular}

Figure 9 displays only a slight difference in the background of the gold XRF signal with increasing Perspex thickness. This is because the source was monoenergetic, and so Compton scatter x-rays are all of a similar energy (the Compton scatter peak was found at $14.6 \mathrm{keV}$ at $90^{\circ}$ detection) and did not contribute to the background of the gold signal. Figure 9 demonstrates that the gold L-XRF lines decrease in intensity with increasing Perspex thickness due to attenuation by the Perspex. All gold signals were found to be above the DL of the system. The predicted and measured reduction in both the gold $\operatorname{L} \alpha$ and $\operatorname{L} \beta$ signals due to overlying thicknesses of Perspex is tabulated in table 2.

\section{Discussion}

Figures 7 and 8 demonstrated that GNP concentrations differing by factors of 20 and 5 could be distinguished by both the step and scan and one-shot XRF imaging systems. Both systems therefore achieved the requirement needed to distinguish tumour from surrounding healthy tissue at GNP concentrations typically expected in vivo. This study has demonstrated the capability of the system to image GNP distribution and to distinguish between GNP concentrations. There is a strong potential that this technique can be extended to quantitative imaging, as this system has already demonstrated ability for quantitative measurement to within $\pm 0.005 \mathrm{mgAu} \mathrm{ml}^{-1}$ at low concentrations (between the detection limit of $0.001 \mathrm{mgAu} \mathrm{ml}^{-1}$ and $0.1 \mathrm{mgAu} \mathrm{ml}^{-1}$ ) (Ricketts et al 2012). 


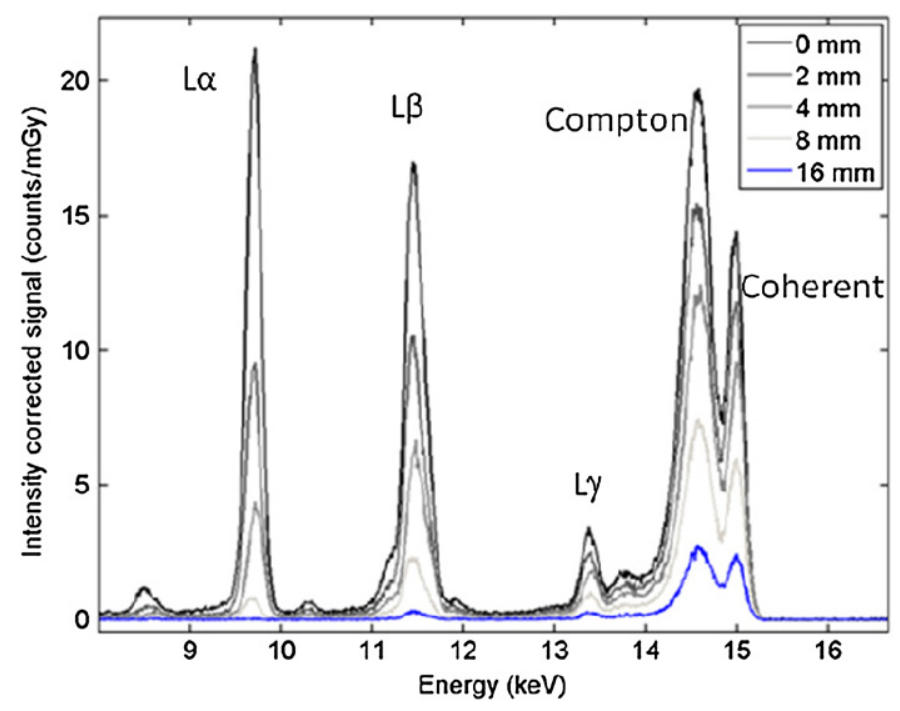

Figure 9. Measured L-XRF spectra of an $8 \mathrm{mgAu} \mathrm{ml}^{-1}$ sample acquired with a range of Perspex thicknesses above the sample.

Figures 7 and 8 also demonstrate that both XRF imaging systems can be used to image GNP concentrations as low as $0.025 \mathrm{mgAu} \mathrm{ml}^{-1}$. Lower concentrations were not tested in this study due to restrictions on acquisition time at the synchrotron source. However it can be inferred from previous detection limit measurements that the step and shoot system may be sensitive to even lower concentrations (Ricketts et al 2012). The addition of polycapillary optic technology will impact on the achievable sensitivity and detection limit in the following ways. Use of the parallel polycapillary optic greatly reduces the collection angle of x-rays emitted from the sample; this decreases the scatter component of the measured spectrum, therefore reducing the background beneath the XRF peak, positively impacting on detection limit. However, the optic also acts to reduce the count rate of the gold XRF signal, which will reduce the sensitivity of the system. The system sensitivity will also be decreased due to transmission losses in the optic. The transmission efficiency of optics is a function of x-ray energy (due to the dependence of the critical angle on energy).

Use of the CDD/parallel optic system improved the achievable spatial resolution of XRF imaging by a factor of 1.6 compared with using the SDD/focusing optic system. There were no differences in efficiency of detection of the gold signal, or achievable count rate between the CDD and SDD. The $\sim 1 \mathrm{keV}$ energy resolution of the CDD was sufficient to distinguish the gold $\mathrm{L} \alpha$ and $\mathrm{L} \beta$ peaks, and could also easily distinguish the scatter peaks and the gold XRF signal. The only effect the degradation in energy resolution would have compared to the SDD is that the detection limit of the system will worsen. A more detailed study in the future is required to quantify the achievable detection limit of the CDD system. Use of the CDD also enabled quicker acquisition times, as it was used to simultaneously collect the XRF spectra for 816 pixels; in order to acquire data for an image with the same number of pixels, the SDD system would have to perform 816 individual scans leading to acquisition times nearly three orders of magnitude greater. 2D images may be acquired over a range of slices in order to construct 3D images of the sample.

The crescent shape seen in the right of the left-hand image in figure 7 is due to attenuation of the primary beam in penetrating the sample. In order to account for beam absorption a scatter 
normalization technique was implemented. The resulting normalized image in log scale is also displayed in figure 7, and shows improved uniformity after correcting for attenuation effects.

At this stage, the imaging system does not provide absolute quantitative information of GNP concentration. In order to achieve this in future system development, the following must be considered.

- Partial volume effect and correction methods to account for this.

- The system must be fully calibrated so that the gold XRF signal at a known beam thickness and solid angle is known.

- Further methods of attenuation correction should be investigated. One possibility is the use of transmission images to measure the attenuation coefficient of each pixel in order to perform the absorption correction.

The predicted and measured effects of overlying thicknesses of Perspex were found to be in good agreement; differences may be attributed to the fact that measurement was not made in narrow beam geometry. As expected the reduction in signal due to 4 (8) $\mathrm{mm}$ overlying Perspex was measured to be $61 \%(84 \%)$ and $77 \%$ (95\%) for the gold $\mathrm{L} \beta$ and $\mathrm{L} \alpha$ signals respectively. Even though the $\mathrm{L} \beta$ signal has a lower fluorescence yield than the $\mathrm{L} \alpha$ peak, it may be beneficial to use the $\mathrm{L} \beta$ peak for analysis of measurements made at depth due to its greater energy. The penetrability of L-XRF makes it sufficient to image GNP distribution in small-animal studies where the GNPs are distributed near the surface of the animal, and a useful tool for imaging 3D in vitro cellular constructs. There are depth limitations to the LXRF imaging technique making it currently unable to image deep tumours. However K-XRF techniques may be implemented for deeper imaging studies; the predicted reduction in gold $\mathrm{K} \alpha \mathrm{XRF}$ signal due to $8 \mathrm{~mm}$ overlying Perspex is only $16 \%$.

Even though K-XRF systems penetrate to greater depths, they cannot achieve sensitivity to low concentrations; a compromise must be made between GNP concentration sensitivity and penetration depth. The L-XRF imaging system we developed demonstrated sensitivity to GNP concentrations up to two orders of magnitude lower than that achieved by the K-XRF XFCT system reported in the literature (Jain et al 2012); even though the fluorescence yields for K-shells is more than a factor of 3 larger than for the L-shells, the photoelectric cross section just above the L-edge of gold $\left(\sigma(11.98 \mathrm{keV})=179.1 \mathrm{~cm}^{2} \mathrm{~g}^{-1}\right)$ is over 21 times greater than that just above the K-edge $\left(\sigma(81.13 \mathrm{keV})=8.3 \mathrm{~cm}^{2} \mathrm{~g}^{-1}\right)$ (calculated from values taken from Chantler et al (2005)). Also, the ratio of photoelectric cross section to total scatter cross section (corresponding to signal to noise ratio) is greater for L-XRF than K-XRF (ratios of 43.4 and 22.0 at the L-edge and K-edge energies respectively). This means that the scatter contribution to the background beneath the gold signal for the K-XRF system is double that for the L-XRF system, making it less sensitive to low concentration measurement.

\section{Conclusions}

A high detecting sensitivity to GNPs can be achieved using XRF techniques, at depths greater than optical modalities currently used. An XRF spectrometer (SDD) coupled to polycapillary lenses was used to define measurement volumes in order to build up pixellated images of GNP-loaded phantoms. The feasibility of performing energy resolved imaging of GNP distribution at concentrations relevant for in vivo and in vitro studies has been demonstrated. GNP concentrations different by a factor of 5 have been shown to be easily resolvable, making this system capable of distinguishing tumour from non-tumour. Further work is required to test the contrast resolution capability of the system further. The L-XRF system can successfully detect GNPs at depths relevant for 3D in vitro cell studies and small-animal studies up to 
centimetre tissue depths, but not currently at depths needed for imaging deep tumours in vivo. Details of phantoms with concentrations as low as $0.025 \mathrm{mgAu} \mathrm{ml}^{-1}$ (the lowest concentration present in the phantom) have been successfully imaged in this study. It can be inferred from previous detection limit measurements that the system will have sensitivity to even lower concentrations. In order to avoid step and scan XRF imaging techniques (potentially requiring long measuring times) the feasibility of excitation of the whole specimen with a wide beam and detection of the fluorescent $\mathrm{x}$-rays with a pixellated energy resolving detector without scanning has been investigated. A parallel polycapillary optic coupled with a CDD has been successfully used to ascertain the position where fluorescence was emitted. Further work is required to investigate methods to perform absolute quantitative imaging. This is challenging, and will involve development of correction procedures for sample attenuation and partial volume effect, and other calibration techniques to gain absolute concentration information.

\section{Acknowledgments}

The authors are indebted to Denzil Booth from University College London Hospital for making the plastic phantoms, and Katherine Holt of the UCL Chemistry Department for her assistance in sample preparation. We would like to thank the beamline scientists at the SYRMEP beamline of the Elettra Synchrotron source, Italy, and the Diamond Light Source, UK, with particular thanks to Kawal Sawhney and Igor Dolbnya for their contribution to set-up and measurements. We are also grateful to Pietro Zambon from the Dipartimento di Elettronica e Informazione, Politecnico di Milano, Italy for developing the custom software used to automatically scan the sample/detector system, and Giuseppe Montemurro for his assistance during the synchrotron measurements.

\section{References}

Alberti R, Grassi N, Guazzoni C and Klatka T 2009 Optimized readout configuration for PIXE spectrometers based on silicon drift detectors: architecture and performance Nucl. Instrum. Methods Phys. Res. A $607458-62$

Andermann G and Kemp J W 1958 Scattered X-rays as internal standards in X-ray emission spectroscopy Anal. Chem. 30 1306-9

Bui C, Confalonieri L and Milazzo M 1989 Use of Compton scattering in quantitative XRF analysis of stained glass Int. J. Radiat. Appl. Instrum. A 40 375-80

Castoldi A, Guazzoni C, Hartmann R and Struder L 2007 Principle and applications of controlled-drift detectors Nucl. Instrum. Methods Phys. Res. A $57979-82$

Castoldi A, Guazzoni C, Pepper K, Gibson A, Griffiths J, Royle G J and Bjeoumikhov A 2011 2D energy-resolved imaging of gold nanoparticle distribution at concentrations relevant for in-vitro studies IEEE Trans. Nucl. Sci. $\mathbf{5 8} 2124-8$

Chantler C, Olsen K, Dragoset R, Chang J, Kishore A, Kotochigova S and Zucker D 2005 X-ray form factor, attenuation and scattering tables (version 2.1) (Gaithersburg, MD: National Institute of Standards and Technology) Gaithersburg, MD, 2005 http://physics.nist.gov/ffast

Chen Q, Li K, Wen S, Liu H, Peng C, Cai H, Shen M, Zhang G and Shi X 2013 Targeted CT/MR dual mode imaging of tumors using multifunctional dendrimer-entrapped gold nanoparticles Biomaterials 34 5200-9

Cheong S-K, Jones B L, Siddiqi A K, Liu F, Manohar N and Cho S H 2010 X-ray fluorescence computed tomography (XFCT) imaging of gold nanoparticle-loaded objects using 110 kVp x-rays Phys. Med. Biol. 55 647-62

Dilmanian F A et al 1997 Single-and dual-energy CT with monochromatic synchrotron x-rays Phys. Med. Biol. 42 371-87

Giljohann D A, Seferos D S, Daniel W L, Massich M D, Patel P C and Mirkin C A 2010 Gold nanoparticles for biology and medicine Angew. Chem. Int. Edn Engl. 49 3280-94

Goodman T, C P Ng and S H Pun 2009 3-D tissue culture systems for the evaluation and optimization of nanoparticlebased drug carriers Bioconjug. Chem. 19 1951-9

Grainger S J, Serna J V, Sunny S, Zhou Y, Deng C X and El-Sayed M E H 2010 Pulsed ultrasound enhances nanoparticle penetration into breast cancer spheroids Mol. Pharm. 15 597-601 
Grassi N, Guazzoni C, Alberti R, Klatka T and Bjeoumikhov A 2010 External scanning micro-PIXE for the characterization of a polycapillary lens: measurement of the collected X-ray intensity distribution Nucl. Instrum. Methods Phys. Res. B 268 1945-8

Hainfeld J F, Dilmanian F A, Zhong Z, Slatkin D N, Kalef-Ezra J A and Smilowitz H M 2010 Gold nanoparticles enhance the radiation therapy of a murine squamous cell carcinoma Phys. Med. Biol. 55 3045-59

Hainfeld J F, O'Connor M J, Dilmanian F A, Slatkin D N, Adams D J and Smilowitz H M 2011 Micro-CT enables microlocalisation and quantification of Her2-targeted gold nanoparticles within tumour regions Br. J. Radiol. 84 526-33

Hainfeld J F, Slatkin D N, Focella T M and Smilowitz H M 2006 Gold nanoparticles: a new x-ray contrast agent Br. J. Radiol. 79 248-53

Hainfeld J F, Slatkin D N and Smilowitz H M 2004 The use of gold nanoparticles to enhance radiotherapy in mice Phys. Med. Biol. 49 N309-15

Hainfeld J F, Smilowitz H M, O’Connor M J, Dilmanian F A and Slatkin D N 2012 Gold nanoparticle imaging and radiotherapy of brain tumors in mice Future Med. 8 1601-9 doi:10.2217/NNM.12.165

Jain S, Hirst DG and O'Sullivan J M 2012 Gold nanoparticles as novel agents for cancer therapy $B r . J$. Radiol. 85 101-13

Jain S et al 2011 Cell-specific radiosensitization by gold nanoparticles at megavoltage radiation energies Int. J. Radiat. Oncol. Biol. Phys. 79 531-9

Jiao P F, Zhou H Y, Chen L X and Yan B 2011 Cancer-targeting multifunctionalized gold nanoparticles in imaging and therapy Curr. Med. Chem. 18 2086-102

Kemp M M and Linhardt R J 2010 Heparin-based nanoparticles WIREs Nanomed. Nanobiotechnol. 2 77-87

Keren S, Zavaleta C, Cheng Z, de la Zerda A, Gheysens O and Gambhir S S 2008 Noninvasive molecular imaging of small living subjects using Raman spectroscopy Proc. Natl Acad. Sci. USA 105 5844-9

Khlebtsov N and Dykman L 2011 Biodistribution and toxicity of engineered gold nanoparticles: a review of in vitro and in vivo studies Chem. Soc. Rev. 40 1647-71

Kim J-K, Seo S-J, Kim K-H, Kim T-J, Chung M-H, Kim K-R and Yang T-K 2010 Therapeutic application of metallic nanoparticles combined with particle-induced X-ray emission effect Nanotechnology 21425102

Kircher M F et al 2012 A brain tumor molecular imaging strategy using a new triple-modality MRI-photoacousticRaman nanoparticle Nature Med. 18 829-34

Kuang Y, Pratx G, Bazalova M, Meng B, Qian J and Xing L 2013 First demonstration of multiplexed x-ray fluorescence computed tomography (XFCT) imaging IEEE Trans. Med. Imaging 32 262-7

Kumakhov M A 1990 Channeling of photons and new X-ray optics Nucl. Instrum. Methods Phys. Res. B $48283-6$

MacDonald C A and Gibson W M 2003 Applications and advances in polycapillary optics X-Ray Spectrom. 32 258-68

Mccarthy J R and Weissleder R 2009 Multifunctional magnetic nanoparticles for targeted imaging and therapy Adv. Drug Deliv. Rev. 60 1241-51

McMahon S J, Prise K M and Currell F J 2012 Comment on 'Implications on clinical scenario of gold nanoparticle radiosensitization in regards to photon energy, nanoparticle size, concentration and location Phys. Med. Biol. 57 287-90

Popovtzer R, Agrawal A, Kotov N A, Popovtzer A, Balter J, Carey T E and Kopelman R 2008 Targeted gold nanoparticles enable molecular CT imaging of cancer Nano Lett. 8 4593-6

Qian X, Peng X-H, Ansari D O, Yin-Goen Q, Chen G Z, Shin D M, Yang L, Young A N, Wang M D and Nie S 2008 In vivo tumor targeting and spectroscopic detection with surface-enhanced Raman nanoparticle tags Nature Biotechnol. 26 83-90

Ricketts K, Castoldi A, Guazzoni C, Ozkan C, Christodoulou C, Gibson A P and Royle G J 2012 A quantitative x-ray detection system for gold nanoparticle tumour biomarkers Phys. Med. Biol. 57 5543-55

Sokolov K, Follen M, Aaron J, Pavlova I, Malpica A, Lotan R and Richards-Kortum R 2004 Real-time vital optical imaging of precancer using anti-epidermal growth factor receptor antibodies conjugated to gold nanoparticles Cancer Res. 63 1999-2004

Wang L, Rath B K, Gibson W M, Kimball J C and MacDonald C A 1996 Performance study of polycapillary optics for hard x rays J. Appl. Phys. 803628

Yang X, Stein E W, Ashkenazi S and Wang L V 2009 Nanoparticles for photoacoustic imaging Wiley Interdiscip. Rev. Nanomed. Nanobiotechnol. $1360-8$

Zaman R T et al 2007 In vivo detection of gold nanoshells in tumors using diffuse optical spectroscopy IEEE J. Sel. Top. Quantum Electron. 13 1715-20

Zhang X-D, Guo M-L, Wu H-Y, Sun Y-M, Ding Y-Q, Feng X and Zhang L-A 2009 Irradiation stability and cytotoxicity of gold nanoparticles for radiotherapy Int. J. Nanomed. 4 165-73 


\section{QUERIES}

\section{Page 1}

Q1

Author: Please provide the e-mail address of the corresponding author.

Q2

Author: Please be aware that the colour figures in this article will only appear in colour in the Web version. If you require colour in the printed journal and have not previously arranged it, please contact the Production Editor now.

\section{Page 2}

Q3

Author: Please check whether the citation of 'Chen et al (2013)' is correct here or you intended to cite 'Hainfeld et al (2012)' instead.

\section{Page 4}

Q4

Author: Figure 1 is not cited in the text. Please check.

\section{Page 9}

Q5

Author: Please check the values '(from $\sim 500 \mu \mathrm{m} 10 \sim 180 \mu \mathrm{m})$ ' in parentheses here. Whether ' 10 ' should be changed to 'to'?

\section{Page 14}

Q6

Author: Please check the details for any journal references that do not have a blue link as they may contain some incorrect information. Pale purple links are used for references to arXiv e-prints.

Q7

Author: Please check whether the author names and journal title are okay as amended in reference 'Goodman et al (2009)'.

\section{Page 15}

Q8

Author: Please check whether the volume number and page range is okay as included in reference 'Hainfeld et al (2012)'.

Q9

Author: Please check whether the journal title in reference 'Kemp and Linhardt (2010)' is okay as amended.

Q10

Author: Please check whether the volume number is okay as included in reference 'Sokolov et al (2004)'. 
Author: Please check whether the journal title, volume number and page range is okay as included in reference 'Yang et al (2009)'.

Q12

Author: Please check whether the journal title is okay as amended in reference 'Zaman et al (2007)'. 